\title{
Binocular Optic Neuritis in an Eight-Year-Old Boy Due to COVID-19 Infection
}

\author{
Hosein Eslamiyeh (iD ${ }^{1}$ and Marjan Jafari ${ }^{2,}{ }^{*}$ \\ ${ }^{1}$ Department of Pediatrics, Shahid Sadoughi University of Medical Sciences, Yazd, Iran \\ ${ }^{2}$ Shahid Sadoughi University of Medical Sciences, Yazd, Iran \\ "Corresponding author: Shahid Sadoughi University of Medical Sciences, Yazd, Iran. Email: dr.marjanjafari@gmail.com
}

Received 2020 December 13; Revised 2021 April 12; Accepted 2021 June 23.

\begin{abstract}
Introduction: Optic neuritis or inflammation of the optic nerve is a frequent cause of acute optic nerve damage in children and adults. Optic neuritis can occur in association with some viruses, such as influenza and mumps viruses. At the end of 2019, a novel coronavirus, known as COVID-19, spread throughout the world. Coronavirus is known to cause optic neuritis in animal models, and several adult cases have been reported up to now, as well. However, to date, no case of optic neuritis has been reported in children with COVID-19.

Case Presentation: The patient was an eight-year-old boy referred to our center, complaining of sudden bilateral and progressive blurring of vision with right-eye preference. He had a history of severe headache for three days in the previous two weeks and slight mood disturbance. On physical examination, bilateral disk swelling and significant loss of vision were detected in his both eyes. Magnetic resonance imaging (MRI) of the brain showed bilateral maxillary sinus thickening with mild optic nerves enlargement with slightly hyperintense $\mathrm{T} 2$ and mild post-contrast enhancement. No pathologic finding was seen in the brain, and cerebrospinal fluid and other immunologic tests were normal. Due to the patient's history of headache and outbreak of COVID-19, a nasopharyngeal reverse transcription-polymerase chain reaction (RT-PCR) test for COVID-19 was requested, and a positive result was obtained, confirming the diagnosis of COVID-19 infection. The optic neuritis was treated with pulsed methylprednisolone and the patients' vision improved.

Conclusions: In this article, we reported an eight-year-old child with optic neuritis and headache in the context of COVID-19. No case of optic neuritis has been reported in children with COVID-19.
\end{abstract}

Keywords: Optic Neuritis, COVID-19, Children

\section{Introduction}

Optic neuritis is a common cause of acute optic nerve damage in children and adults (1). Diagnosis of optic neuritis is based on a set of signs and symptoms. It usually starts with pain in eye movements and is associated with acute vision loss (2,3). Different forms of optic neuropathy, including optic neuritis, have been reported in association with infectious agents. Optic neuritis can occur in association with some viruses, such as influenza virus, mumps virus, cytomegalovirus, and Epstein-Barr virus (4).

At the end of 2019, a novel coronavirus, known as COVID-19, spread throughout the world. The World Health Organization declared the outbreak a public health emergency of international concern in January 2020 (5). The most common symptoms of coronavirus disease are fever, dry cough, and shortness of breath (6). Although the most common symptom of COVID-19 is pneumonia, some patients experience other major organ involvements. One of the most involved organs is the nervous system. Both central and peripheral nervous system diseases have been reported following COVID-19.

As known, COVID-19 has neuroinvasive potential given that one-third of adult patients have diverse neurological manifestations $(6,7)$. Among children, COVID-19related neurological manifestations have been reported in some articles, but the number of articles is limited. The most common complications reported in children are headache, positive meningeal signs (meningismus), and altered level of consciousness (8). However, to date, no case of optic neuritis has been reported in children with COVID19. 


\section{Case Presentation}

During the outbreak of the COVID-19 pandemic, an eight-year-old boy was referred to our center complaining of a sudden decrease in vision and bilateral and progressive blurring of vision with right-eye preference. He also had a frontal headache for two weeks, which was more severe in the first three days, which then decreased spontaneously. The headache was more severe in the morning but did not awake the patient. The patient experienced a weight loss of about three kilograms and had slight mood disturbance during the previous two weeks. The patient had no history of eye trauma or exposure to toxins and did not take any medications. He was the second child of the family and was born by normal vaginal delivery. The case had completed all vaccinations required in eight years, and his growth and development were in the normal range. His parents were not relatives, and they did not have a family history of special diseases. The family and children did not follow health and quarantine protocols.

On initial examination, the patient's heart rate was 90, respiratory rate was 20 , and blood oxygen saturation was 97\%. The patient had no fever. On ophthalmologic examination, he had no conjunctivitis. His eye movements were normal and not painful, but he had color blindness in the Ishihara test with a red-green color defect. On ocular cranial nerve examinations, in general, the Marcus Gunn sign was seen, and bilateral disk swelling was observed on fundoscopy. Visual acuity (VA) was 2/10 in the right eye and 4/10 in the left one, so the diagnosis of optic neuritis was established. Other cranial nerve and neurological examinations, including deep tendon reflexes (DTR) and bilateral plantar reflexes, were normal. The force of four limbs was $5 / 5$, and no paresthesia or sensory disturbances were detected.

For more investigation, necessary laboratory tests were requested and the results were as follows:

CBCdiff: $(\mathrm{WBC}=8600 \mathrm{cmm}$, Neut $=50.5 \%$, Lymph $=$ $36.5 \%, \mathrm{Hb}=13.4 \mathrm{~g} / \mathrm{dL}, \mathrm{Plt}=346000 \mathrm{~mL}), \mathrm{TSH}=3.3 \mathrm{mIU} / \mathrm{L}, \mathrm{CRP}$ $=+$, Anti ds DNA $=0.23 \mathrm{IU} / \mathrm{mL}, \mathrm{ANA}=0.21 \mathrm{U} / \mathrm{mL}, \mathrm{ESR}=14$ $\mathrm{mm} / \mathrm{h}$.

In order to rule out demyelinating disorders, brain and cervical spinal MRIs and lumbar puncture (LP) were performed. Brain MRI showed mucosal thickening in maxillary and ethmoid sinuses and mild optic nerves enlargement with hyperintensities in T2 (Figure 1) and postcontrast enhancement (Figure 2). No abnormality was seen in the brain and cervical parenchyma before and after the injection of contrast. So, sinus venous thrombosis was rejected and optic nerve swelling was established.
CSF analysis was in normal range: $(\mathrm{WBC}=0, \mathrm{RBC}=0$, $\mathrm{Glu}=95 \mathrm{mg} / \mathrm{dL}, \mathrm{LDH}=41$, Pro $=25 \mathrm{mg} / \mathrm{dL}, \mathrm{CSF}$ pressure $=16$ $\mathrm{cmH}_{2} \mathrm{O}$ ).

In addition, it was observed that the oligoclonal band in cerebro spinal fluid (CSF) was in the normal range, and serum anti-NMO (neuromyelitis optica) was negative. Therefore, demyelinating disorders, such as multiple sclerosis and neuromyelitis optica, were ruled out.

Considering his history, severe headache, and loss of weight, as well as the outbreak of COVID-19, the nasopharyngeal RT-PCR test for COVID-19 was requested and surprisingly, a positive result was obtained. He was quarantined due to the diagnosis of optic neuritis in the context of COVID-19. Following the diagnosis of optic neuritis, the patient was treated with pulsed methylprednisolone. Having received the medication, his vision improved significantly. Eventually, visual acuity became 7/10 in the right eye and $8 / 10$ in the left one.

\section{Discussion}

Optic neuritis is a common cause of acute optic nerve injury in children and adults (1). Diagnosis of optic neuritis is based on a set of signs and symptoms. It usually starts with pain with eye movements in one or both eyes and is associated with acute vision loss. However, it is often bilateral in children. The main differential diagnosis of optic neuritis is idiopathic intracranial hypertension (IIH). However, IIH can be ruled out by history, physical examination, and paraclinical findings. There is no loss of visual acuity in IIH, and optic nerve enhancement should not be observed in MRI after post-contrast injection. However, CSF pressure is significantly raised in $\mathrm{IIH}$, and it is usually normal in optic neuritis. In addition to the significant loss of visual acuity in our patient, its CSF pressure was in the normal range, and his MRI showed optic nerve swelling with post-contrast enhancement. One-third of patients with optic neuritis have light edematous $\operatorname{discs}(2,3)$. Optic neuritis in children has specific clinical symptoms and fortunately has a better prognosis than in adults. Viruses are the main cause of optic neuritis in children, but the main concern of physicians is the relationship between optic neuritis and demyelinating disorders such as multiple sclerosis (9).

There are several causes for optic nerve inflammation, including autoimmunity, infection, granulomatous disease, paraneoplastic disorders, and myelin depletion (1). A wide range of viral, bacterial, parasitic, and fungal agents, such as herpes virus, influenza virus, varicella-zoster virus, and cytomegalovirus, can cause optic neuritis, with different symptoms. The appropriate clinical diagnosis of the 


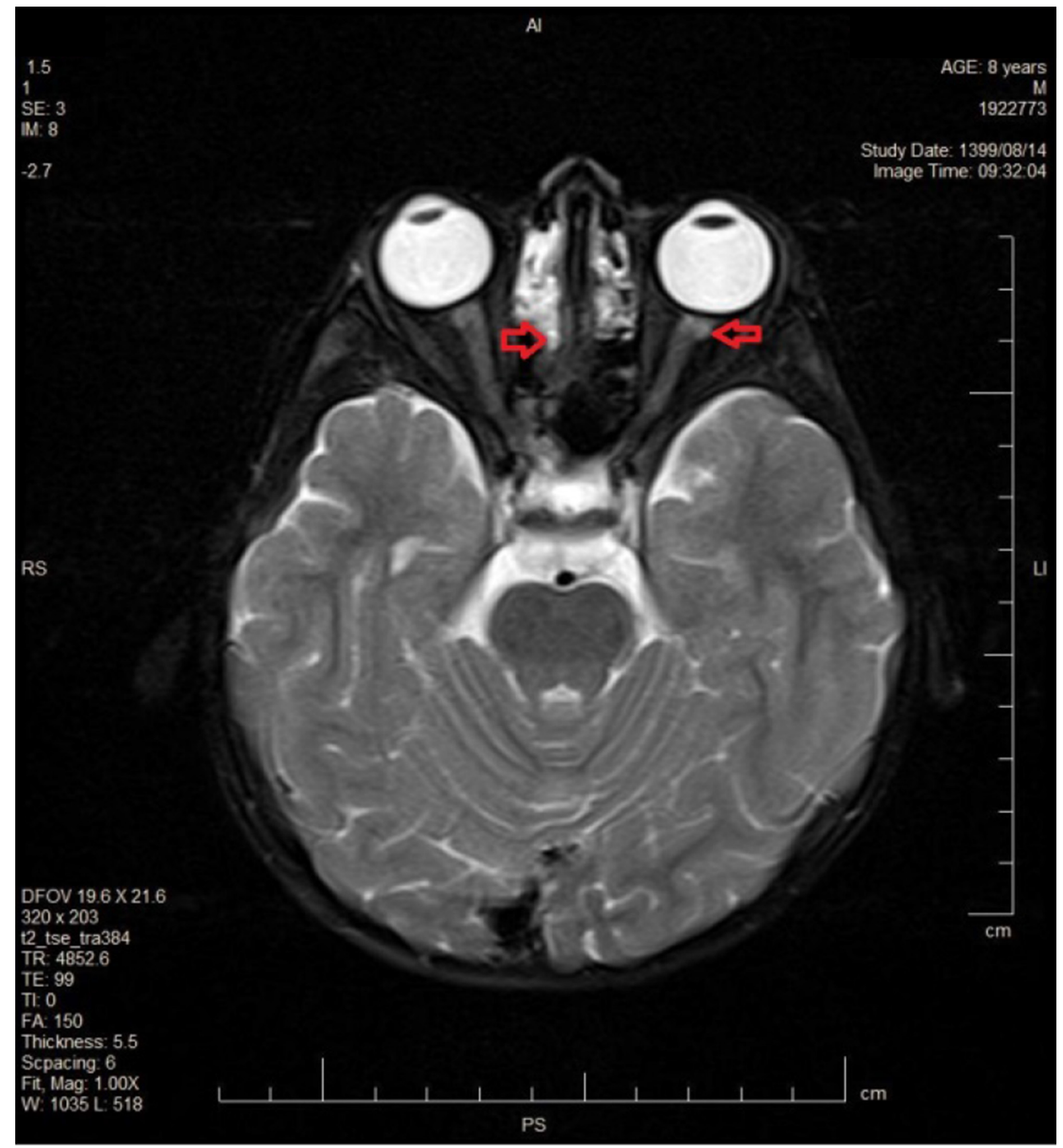

Figure 1. T2-weighted magnetic resonance imaging showing ethmoidal sinus fullness and hyperintensities in the anterior part of the optic nerve with mild enlargement

cause of the disease is based on epidemiological data, underlying disease, systemic signs and symptoms, the pattern of nerve injury, and visual signs that can be confirmed by imaging and laboratory findings. Some of the infectious agents are treatable. Corticosteroids with other specific drugs are used to treat it, but the response to treatment is variable and depends on the cause of the disease (4). Corticosteroids are especially used to treat acute viral neuritis due to their anti-inflammatory effects (3).

Severe acute respiratory syndrome coronavirus 2 (SARS-CoV-2) enters the nervous system through retrograde and anterograde transmission. Besides, SARS-CoV-2 


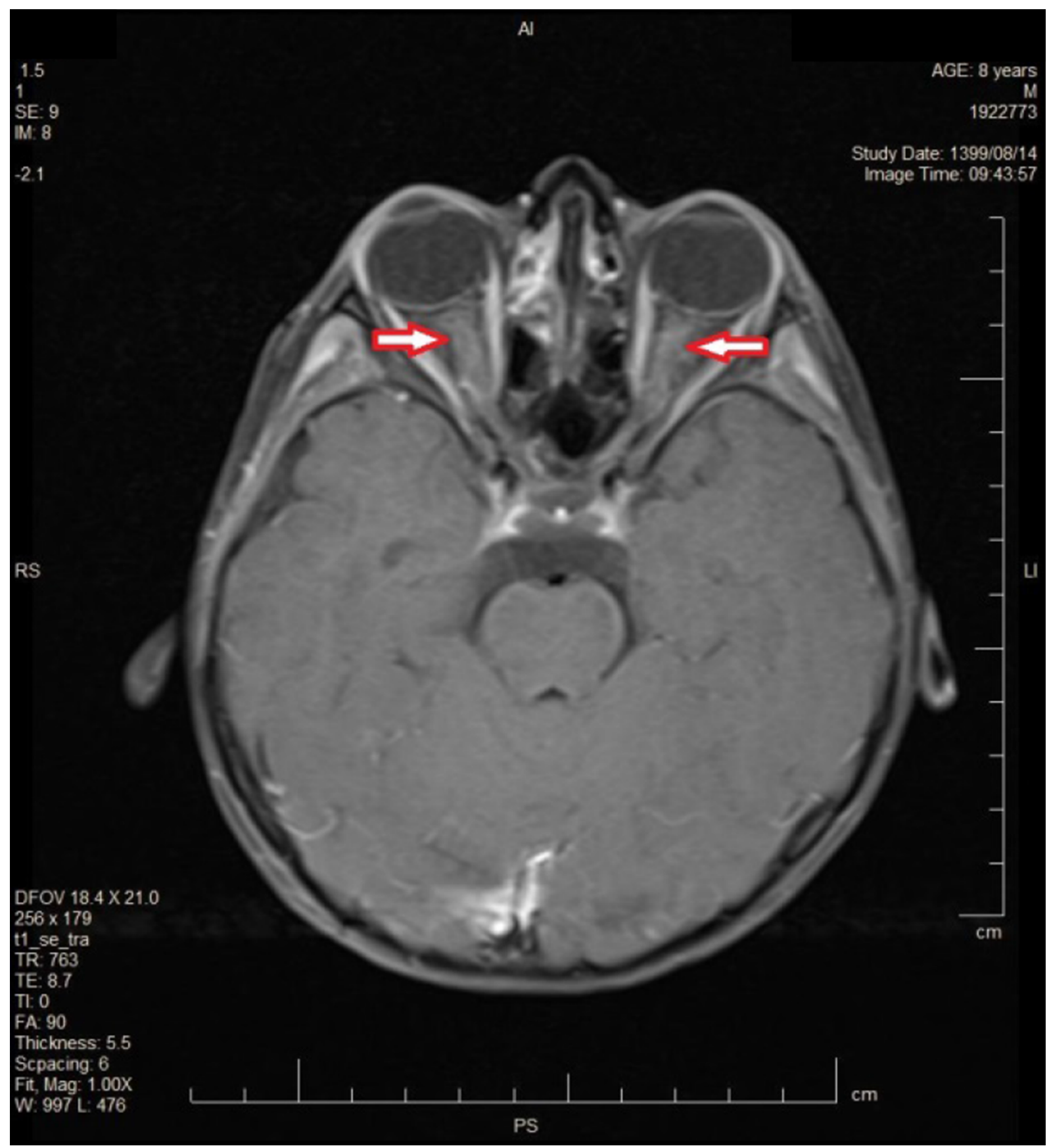

Figure 2. T1-weighted magnetic resonance imaging with contrast injection and fat suppression showing post-contrast enhancement in both optic nerves

invades the olfactory bulb via TMPRSS2 and ACE2 receptors. Additionally, the extracellular vesicles (EVs) of the olfactory membrane can transfer the virus independent of ACE2 receptors. Also, the virus can enter the central nervous system (CNS) through the trigeminal and vagus nerves. Furthermore, the virus can enter the CNS via the bloodstream (10). Among newborns and children, COVID-19-related neurological manifestations have been relatively rare; however, reports of neurodegenerative dysfunction are increasing in this age range (11). 
Both CNS and PNS diseases have been reported following COVID-19 (7). Depressed levels of consciousness, ataxia, and seizures are more common in severely ill patients (11). Among the neurological symptoms of COVID-19 in children, central demyelinating diseases and GuillainBarre syndrome have also been reported rarely following the viral infection (12). In a study on 187 children, 64 had neurological involvement, among which headaches, positive meningeal signs (meningismus), and altered levels of consciousness were most frequent (8). Our patient had three days of severe headache as the first manifestation of the disease, which gradually subsided. In our case, slight mood disturbance was also reported by his mother, which could be due to viral encephalopathy. Consequently, the patient experienced visual loss and optic neuritis. In order to rule out important demyelinating disorders, such as neuromyelitis optica and multiple sclerosis, several investigations were performed, and all of them were negative. Due to the outbreak of COVID-19, a nasopharyngeal RT-PCR test for COVID-19 was requested for him, and the result was positive. Fortunately, there is less evidence that COVID-19 triggers central demyelinating diseases in children (11).

Coronavirus is a known cause of retinitis and optic neuritis in animal models; several adult cases have been also reported up to now $(13,14)$. However, to date, there is no report of optic neuritis in the pediatric population, and our case can be the first report in this regard.

\section{Acknowledgments}

We would like to appreciate the patient and his family for contributing to the treatment and follow-up of the patient.

\section{Footnotes}

Authors' Contribution: Definite diagnosis of the case and critical revision of the manuscript for important intellectual content: Hosein Eslamiyeh. Contributing to case management and writing the article: Marjan Jafari.

Conflict of Interests: The authors declare that there is no conflict of interest.

Ethical Approval: The ethical approval code was IR.SSU.MEDICINE.REC.1399.293.

Funding/Support: We have no supporters.

Informed Consent: Written informed consent was obtained.

\section{References}

1. Bennett JL. Optic Neuritis. Continuum. 2019;25(5):1236-64. doi: 10.1212/con.0000000000000768.

2. Wilhelm $\mathrm{H}$, Schabet $\mathrm{M}$. The diagnosis and treatment of optic neuritis. Deutsches Ärzteblatt Int. 2015;112(37):616.

3. Gal RL, Vedula SS, Beck R. Corticosteroids for treating optic neuritis. Cochrane Database Syst Rev. 2015;(8). CD001430. doi: 10.1002/14651858.CD001430.pub4. [PubMed: 26273799]. [PubMed Central: PMC4730547].

4. MadaniNeishaboori A, Moshrefiaraghi D, Mohamed Ali K, Toloui A, Yousefifard M, Hosseini M. Central Nervous System Complications in COVID-19 Patients; a Systematic Review and Meta-Analysis based on Current Evidence. Arch Acad Emerg Med. 2020;8(1). e62. [PubMed: 33134959]. [PubMed Central: PMC7587989].

5. Ellul M, Benjamin L, Singh B, Lant S, Michael B, Kneen R, et al. Neurological Associations of COVID-19. SSRN Electron J. 2020. doi: 10.2139/ssrn.3589350.

6. Chen TH. Neurological involvement associated with COVID19 infection in children. J Neurol Sci. 2020;418:117096. doi: 10.1016/j.jns.2020.117096. [PubMed: 32823135]. [PubMed Central: PMC7423535].

7. Perez-Cambrodi RJ, Gomez-Hurtado Cubillana A, Merino-Suarez ML, Pinero-Llorens DP, Laria-Ochaita C. Optic neuritis in pediatric population: a review in current tendencies of diagnosis and management. J Optom. 2014;7(3):125-30. doi: 10.1016/j.optom.2013.12.008. [PubMed: 25000867]. [PubMed Central: PMC4087179].

8. Khairallah M, Kahloun R, Abroug N, Ksiaa I, Mahmoud A, Zeghidi H, et al. Infectious optic neuropathies: a clinical update. Eye Brain. 2015. doi: 10.2147/eb.s69173.

9. Beghi E, Feigin V, Caso V, Santalucia P, Logroscino G. COVID-19 Infection and Neurological Complications: Present Findings and Future Predictions. Neuroepidemiology. 2020;54(5):364-9. doi: 10.1159/000508991. [PubMed: 32610334]. [PubMed Central: PMC7445369].

10. Christy A. COVID-19: A Review for the Pediatric Neurologist. J Child Neurol. 2020;35(13):934-9. doi: 10.1177/0883073820939387. [PubMed: 32660309].

11. Stafstrom CE, Jantzie LL. COVID-19: Neurological Considerations in Neonates and Children. Children (Basel). 2020;7(9). doi: 10.3390/children7090133. [PubMed: 32927628]. [PubMed Central: PMC7552690].

12. Seah I, Agrawal R. Can the Coronavirus Disease 2019 (COVID-19) Affect the Eyes? A Review of Coronaviruses and Ocular Implications in Humans and Animals. Ocul Immunol Inflamm. 2020;28(3):391-5. doi: 10.1080/09273948.2020.1738501. [PubMed: 32175797]. [PubMed Central: PMC7103678]

13. Beck RW, Cleary PA, Anderson MJ, Keltner JL, Shults WT, Kaufman DI, et al. A randomized, controlled trial of corticosteroids in the treatment of acute optic neuritis. The Optic Neuritis Study Group. N Engl J Med.1992;326(9):581-8. doi: 10.1056/NEJM199202273260901. [PubMed: 1734247].

14. World Health Organization. Director-General's remarks at the media briefing on 2019-nCoV. 2020, [cited 11 Feb 2020]. Available from: http://www.who.int/dg/speeches/detail/who-director-general-sremarks-at-the-media-briefing-on-2019-ncov-on-11-february2020. 